Petra Steinacker, PhD

Elisa Semler, Dipl-Psych

Sarah Anderl-Straub, PhD

Janine Diehl-Schmid,

MD

Matthias L. Schroeter, MD, PhD

Ingo Uttner, $\mathrm{PhD}$

Hans Foerstl, MD

Bernhard

Landwehrmeyer, MD

Christine A.F. von Arnim, MD

Jan Kassubek, MD

Patrick Oeckl, PhD

Hans-Jürgen Huppertz, MD

Klaus Fassbender, MD

Klaus Fliessbach, MD

Johannes Prudlo, MD

Carola Roßmeier, MD

Johannes Kornhuber, MD

Anja Schneider, MD

Alexander E. Volk, MD

Martin Lauer, MD

Adrian Danek, MD

Albert C. Ludolph, MD

Markus Otto, MD

On behalf of the FTLDc

Study Group

Correspondence to

Dr. Otto:

markus.otto@uni-ulm.de

Supplemental data at Neurology.org

\section{Neurofilament as a blood marker for diagnosis and monitoring of primary progressive aphasias}

\section{$\underline{\underline{\underline{\underline{A}}}}$}

\section{ABSTRACT}

Objective: To assess the utility of serum neurofilament for diagnosis and monitoring of primary progressive aphasia (PPA) variants.

Methods: We investigated neurofilament light chain (NF-L) levels in blood of 99 patients with PPA (40 with nonfluent variant PPA [nfvPPA], 38 with semantic variant PPA [svPPA], 21 with logopenic variant PPA [IVPPA]) and compared diagnostic performance with that reached by CSF $N F-L$, phosphorylated neurofilament heavy chain (pNF-H), $\beta$-amyloid $\left(A \beta_{1-42}\right)$, tau, and phosphorylated tau. The longitudinal change of blood NF-L levels was measured and analyzed for correlation with functional decline and brain atrophy.

Results: Serum NF-L is increased in PPA compared to controls and discriminates between nfvPPA/svPPA and IvPPA with $81 \%$ sensitivity and $67 \%$ specificity (cutoff $31 \mathrm{pg} / \mathrm{mL}$ ). CSF $N F-L$, pNF-H, tau, phosphorylated tau, and $A \beta_{1-42}$ achieved similar performance, and pNF-H was the only marker for discrimination of nfvPPA from svPPA/lvPPA. In most patients with nfvPPA and svPPA, but not IVPPA, serum NF-L increased within follow-up. The increase correlated with functional decline and progression of atrophy of the left frontal lobe of all patients with PPAs and the right middle frontal gyrus of patients with nfvPPA and svPPA.

Conclusions: Blood level of NF-L can aid the differential diagnosis of PPA variants, especially in combination with CSF pNF-H. Because serum NF-L correlates with functional decline and atrophy in the disease course, it qualifies as an objective disease status marker. Extended follow-up studies with cases of known neuropathology are imperative.

Classification of evidence: This study provides Class I evidence that in patients with PPA, blood levels of NF-L can distinguish the logopenic variant from the nonfluent/agrammatic and semantic variants. Neurology ${ }^{\circledR}$ 2017;88:961-969

\section{GLOSSARY}

$\mathbf{A} \boldsymbol{\beta}=\beta$-amyloid $\mathbf{C D R}=$ Clinical Dementia Rating; $\mathbf{F T L D}=$ frontotemporal lobar degeneration; IvPPA = logopenic variant of primary progressive aphasia; MMSE = Mini-Mental State Examination; NF-L = neurofilament light chain; nfvPPA = nonfluent/agrammatic variant of primary progressive aphasia; $\mathbf{P N F - H}=$ phosphorylated neurofilament heavy chain; PPA = primary progressive aphasia; $\mathbf{p}$-tau $=$ phosphorylated tau; $\mathbf{R O C}=$ receiver-operating characteristics; $\mathbf{s v P P A}=$ semantic variant of primary progressive aphasia; $\mathbf{v} \mathbf{~ = ~ b a s e l i n e ~ v i s i t ; ~} \mathbf{v} \mathbf{2}=$ follow-up visit.

Primary progressive aphasias (PPAs) are a heterogeneous group of progressive language disorders within the clinical spectrum of frontotemporal lobar degeneration (FTLD). According to recently revised diagnostic criteria, ${ }^{1} 3$ variants are distinguished on the basis of the profile of language impairment, distribution of atrophy, and likelihood of underlying neuropathology: a nonfluent/agrammatic variant (nfvPP), a semantic variant (svPA), and a logopenic variant (lvPPA).

\footnotetext{
From the Department of Neurology (P.S., E.S., S.A.-S., I.U., B.L., C.A.F.v.A., J. Kassubek, P.O., A.C.L., M.O.), University of Ulm; Department of Psychiatry and Psychotherapy (J.D.-S., H.F., C.R.), Technical University of Munich; Clinic for Cognitive Neurology (M.L.S.), University Clinic Leipzig and Max Planck Institute for Human Cognitive and Brain Sciences, Germany; Swiss Epilepsy Center (H.-J.H.), Zürich, Switzerland; Department of Neurology (K. Fassbender), Saarland University, Homburg; Department of Psychiatry and Psychotherapy (K. Fliessbach), University of Bonn and DZNE; Department of Neurology (J.P.), University of Rostock and German Center for Neurodegenerative Diseases; Department of Psychiatry and Psychotherapy (J. Kornhuber), Friedrich-Alexander-University of Erlangen-Nuremberg, Erlangen; Department of Psychiatry and Psychotherapy (A.S.), University of Göttingen; Institute of Human Genetics (A.E.V.), University Medical Center HamburgEppendorf, Hamburg; Department of Psychiatry and Psychotherapy (M.L.), University of Würzburg; and Department of Neurology (A.D.), Ludwig-Maximilians-University Munich, Germany. FTLDc Coinvestigators are listed at Neurology.org.

Go to Neurology.org for full disclosures. Funding information and disclosures deemed relevant by the authors, if any, are provided at the end of the article.
} 
There is a need for neurochemical markers for PPA differential diagnosis, severity, progression, and molecular pathology that, together with neuropsychological and imaging approaches, aid patient stratification and allow monitoring in future etiology-specific treatments. ${ }^{2}$

Neurofilament light chain (NF-L), a marker of neurodegeneration, is increased in the CSF of patients with dementia, including frontotemporal dementia, correlating with disease severity and outcome. ${ }^{3-5}$ Recently, NF-L was shown to be also increased in the blood of patients with FTLD compared to controls, correlating with frontal lobe atrophy. ${ }^{6}$

We hypothesized that blood NF-L level can aid the discrimination between PPA subtypes and represents a correlate for disease progression.

In longitudinally collected serum samples from patients with nfvPPA, svPPA, and lvPPA, we analyzed NF-L, compared the diagnostic performance at baseline with that of CSF markers (NF-L, phosphorylated NF heavy chain $[\mathrm{pNF}-\mathrm{H}]$, tau, phosphorylated tau [p-tau], $\beta$-amyloid $\left.\left[A \beta_{1-42}\right]\right)$, and examined the course of NF-L in correlation to functional decline and brain atrophy.

METHODS The primary research questions were the following: Do serum NF-L levels discriminate between PPA variants (Class I level of evidence), and are longitudinal serum NF-L levels suitable to monitor disease progression in PPA (Class I level of evidence)?

Patients. Ninety-nine patients with PPA were included in the study, of whom 37 were examined longitudinally. As a control group, 35 healthy participants without a neurodegenerative disease and without acute or chronic inflammation of the brain were included, of whom 10 attended twice.

Standard protocol approvals, registrations, and patient consents. All participants were examined between April 2011 and December 2015 within the German FTLD consortium, a quality-controlled, monitored, multicenter initiative (www. ftld.de). ${ }^{7}$ The study was approved by the local ethics committees of the centers involved in Ulm, Munich, Leipzig, Rostock, Bonn, Göttingen, Homburg, Würzburg, and Erlangen (proposal number at central study center at University of Ulm: 39/11, March 8, 2011). Each patient, participant, caregiver, or legal representative provided written informed consent for the study according to institutional guidelines.

Patients with FTLD were examined at 2 visits ( $\mathrm{v} 1=$ baseline and $\mathrm{v} 2=$ follow-up) with an interval of 12 months. CSF samples taken by lumbar puncture at $\mathrm{v} 1$ were provided by 81 patients.

Table 1 gives demographic characteristics and clinical assessments of the patient groups.

Patients with PPA underwent standardized clinicalneurological and routine laboratory examinations. Patients with PPA met standard diagnostic criteria according to clinical, neuropsychological, and imaging results ${ }^{1}$ without knowledge of fluid marker levels. The comprehensive neuropsychological assessment was done according to the German FTLD consortium protocol, covering all relevant cognitive domains with a focus on language and executive functions. ${ }^{8}$

Severity of symptoms was classified by functional assessment and global rating scales (Mini-Mental State Examination [MMSE], ${ }^{9}$ Clinical Dementia Rating $[\mathrm{CDR}],{ }^{10}$ and FTLD$\mathrm{CDR}^{11}$ ). Disease progression rate at baseline was calculated by dividing the change in the functional rating by the months since perceived disease onset. Progression rate between v1 and v2 was calculated analogously.

Genetic analysis of C9orf72 hexanucleotide repeat in $72 \mathrm{pa}-$ tients and of the MAPT and GRN genes in 27 patients with a positive familial history revealed no mutations (details are available on request).

MRI and volumetric analysis. The $3 \mathrm{~T}$ MRI data, including 3-dimensional T1 magnetization-prepared rapid gradient-echo sequences, were acquired using the same standardized protocol in all study centers, with regular phantom-based monitoring of scanner performance at each site. For 32 patients with PPA and 10 controls, imaging data at baseline examination were available. For 22 patients with PPA and 10 control participants, MRI was recorded at $\mathrm{v} 1$ and $\mathrm{v} 2$. To determine the volumes of brain structures, we applied atlas-based volumetry, a fully automated and objective method for volumetric analysis of individual patients, using algorithms of the Statistical Parametric Mapping 12 software (Wellcome Trust Centre for Neuroimaging, London, UK; www.fil.ion.ucl.ac.uk/spm). As previously described, ${ }^{12,13}$ the individual brain is segmented into gray matter, white matter, and CSF compartments, and the resulting tissue component images are mapped into a template space by means of high-dimensional elastic registration. Then, in the same space, predefined regions of interest derived from probabilistic brain atlases such as the LONI Probabilistic Brain Atlas $^{14}$ and the Harvard-Oxford atlas of subcortical structures ${ }^{15}$ are used to extract regional brain volumes. Atlas-based volumetry has been successfully used in a variety of cross-sectional and longitudinal studies, ${ }^{13,16,17}$ and a recent study showed that the intrascanner variability of volumetric results is $<1 \%$ for the majority of structures investigated. ${ }^{18}$

Following a meta-analysis that validated the current PPA imaging criteria, ${ }^{19}$ gray matter volumes of 32 brain areas with a focus on frontal and temporal areas were included.

Laboratory markers. Serum was extracted from blood, divided into aliquots, and stored within 2 hours at $-80^{\circ} \mathrm{C}$ until analysis. CSF was obtained by lumbar puncture and processed likewise. All analyses were performed in a blinded manner. Commercially available ELISAs were used to measure CSF NF-L (IBL, Hamburg, Germany), pNF-H (Biovendor, Heidelberg, Germany), tau, and p-tau (Fujirebio, Hanover, Germany) according to the manufacturers' instructions. Mean coefficients of variation of the assays were $<20 \%$. NF-L in serum samples was measured with an electrochemiluminescence immunoassay as described previously with an interassay coefficient of variation $<10.8 \%{ }^{20,21}$ Analyses of longitudinal NF-L measures were applied to original and normalized data. For normalization, the change in concentration was divided by the mean concentration at baseline of all patients of the group analyzed.

Statistical analysis. Statistical analysis was performed with GraphPad Prism 5.0. Because NF-L measures did not pass Shapiro-Wilk normality test $(p<0.0001$ for the PPA cohort 


\begin{tabular}{|c|c|c|c|c|c|c|c|c|c|c|c|c|}
\hline \multirow[t]{3}{*}{ Table 1} & $\begin{array}{l}\text { Its' demographic } \\
\text { ssive aphasia an }\end{array}$ & $\begin{array}{l}\text { haracteristics, } \\
\text { in controls }\end{array}$ & unctional and & obal rating s & cale assessment & and available & RI scans in the & nonfluent/ag & rammatic, sema & ntic, and logop & enic variant o & primary \\
\hline & \multicolumn{2}{|l|}{ All PPA } & \multicolumn{2}{|l|}{ nfvPPA } & \multicolumn{2}{|l|}{ svPPA } & \multicolumn{2}{|l|}{ nfvPPA + svPPA } & \multicolumn{2}{|l|}{ IvPPA } & \multicolumn{2}{|l|}{ Controls } \\
\hline & v1 & v2 & v1 & v2 & v1 & v2 & v1 & v2 & $\mathrm{v} 1$ & v2 & v1 & v2 \\
\hline Sex, F/M & $49 / 50$ & $21 / 16$ & $23 / 17$ & $10 / 7$ & $18 / 20$ & $11 / 4$ & $41 / 37$ & $21 / 11$ & $8 / 13$ & $0 / 5$ & $19 / 16$ & $5 / 5$ \\
\hline Age, $y^{a}$ & $66.3(45-80)$ & $66.4(50-79)$ & $70.4(52-80)$ & $70.2(53-80)$ & $63.8(45-79)$ & $62.5(58-73)$ & $65.3(45-80)$ & 66.5 (53-79) & $68.6(49-78)$ & $67.7(65-75)$ & $63.6(37-75)$ & $\begin{array}{l}64.8 \\
(38-76)\end{array}$ \\
\hline Age at onset, $y$ & 64 (44-78) & $60(47-76)$ & $68(47-78)$ & $64(47-76)$ & $60(44-75)$ & $57(49-68)$ & $64(44-78)$ & 61 (47-76) & $64(46-72)$ & $60(55-72)$ & & \\
\hline Disease duration, $y$ & $2.8(0.2-19.9)$ & $3.3(1-18.7)$ & $2.2^{b}(0.2-8.9)$ & $3.2(1.8-6.2)$ & $3.0^{\circ}(0.6-19.9)$ & $4.6(1-7.7)$ & $2.6(0.2-19.9)$ & $3.3(1-7.7)$ & $3.3^{d}(0.5-17.7)$ & $7.3(2.4-18.7)$ & & \\
\hline$\underset{n}{\text { CDR sob }}$ & $\begin{array}{l}2.5(0-17) \\
89\end{array}$ & $\begin{array}{l}4.3(0.5-16) \\
37\end{array}$ & $\begin{array}{l}2.0(0-17) \\
35\end{array}$ & $\begin{array}{l}6.25(1-13) \\
12\end{array}$ & $\begin{array}{l}3.5(0-11) \\
32\end{array}$ & $\begin{array}{l}5.75(2-16) \\
10\end{array}$ & $\begin{array}{l}2.5(0-17) \\
71\end{array}$ & $\begin{array}{l}5.3(0.5-16) \\
22\end{array}$ & $\begin{array}{l}2.8(0-16) \\
18\end{array}$ & $\begin{array}{l}2.5(1-6) \\
4\end{array}$ & & \\
\hline $\begin{array}{l}\text { FTLD-CDR sob } \\
\mathbf{n}\end{array}$ & $\begin{array}{l}4.5(0.5-23) \\
85\end{array}$ & $\begin{array}{l}7(1-22) \\
37\end{array}$ & $\begin{array}{l}4.0(0.5-23) \\
35\end{array}$ & $\begin{array}{l}9.0(1-18) \\
13\end{array}$ & $\begin{array}{l}4.8(1.5-14) \\
32\end{array}$ & $\begin{array}{l}9.25(4-21) \\
10\end{array}$ & $\begin{array}{l}4.5(0.5-23) \\
71\end{array}$ & $\begin{array}{l}8(1-22) \\
23\end{array}$ & $\begin{array}{l}5.0(1-21) \\
18\end{array}$ & $\begin{array}{l}4.25(3-11) \\
4\end{array}$ & & \\
\hline$\underset{n}{\text { MMSE }}$ & $\begin{array}{l}24(3-30) \\
94\end{array}$ & $\begin{array}{l}23(3-30) \\
37\end{array}$ & $\begin{array}{l}25(10-30) \\
37\end{array}$ & $\begin{array}{l}20(3-29) \\
13\end{array}$ & $\begin{array}{l}24(3-30) \\
33\end{array}$ & $\begin{array}{l}21.5(4-28) \\
12\end{array}$ & $\begin{array}{l}24 \text { (3-30) } \\
74\end{array}$ & $\begin{array}{l}22.5(3-30) \\
34\end{array}$ & $\begin{array}{l}24.0(6-29) \\
20\end{array}$ & $\begin{array}{l}25.0(11-28) \\
4\end{array}$ & & \\
\hline MRI, n & 32 & 22 & 17 & 12 & 8 & 7 & 25 & 19 & 7 & 3 & 10 & 10 \\
\hline $\begin{array}{l}\text { NF-L serum, pg/mL } \\
n\end{array}$ & $\begin{array}{l}49.1(7.6-304) \\
99\end{array}$ & $\begin{array}{l}72.7(9.8-411) \\
37\end{array}$ & $\begin{array}{l}54.2 \\
(10.2-304) \\
40\end{array}$ & $\begin{array}{l}77.3 \\
(18-147) \\
17\end{array}$ & $\begin{array}{l}52.7(7.6-183) \\
38\end{array}$ & $\begin{array}{l}97.3 \\
(23.9-411) \\
15\end{array}$ & $\begin{array}{l}54.2(7.6-304) \\
78\end{array}$ & $\begin{array}{l}77.3 \\
(18-411) \\
32\end{array}$ & $\begin{array}{l}26(7.6-219) \\
21\end{array}$ & $\begin{array}{l}28.9(9.8-71) \\
5\end{array}$ & $\begin{array}{l}18.4 \\
(2.1-75.6) \\
35\end{array}$ & $\begin{array}{l}15.0 \\
(6.7-41.4) \\
10\end{array}$ \\
\hline $\begin{array}{l}\text { NF-L CSF, pg/mL } \\
n\end{array}$ & $\begin{array}{l}2,653 \\
(502-9,452) \\
64\end{array}$ & & $\begin{array}{l}3,160 \\
(1,158-7,474) \\
25\end{array}$ & & $\begin{array}{l}2,936 \\
(828-9,452) \\
26\end{array}$ & & $\begin{array}{l}2,992 \\
(828-9,452) \\
51\end{array}$ & & $\begin{array}{l}1,501 \\
(502-2,859) \\
13\end{array}$ & & $\begin{array}{l}624 \\
(302-1,528) \\
5\end{array}$ & \\
\hline $\begin{array}{l}\mathrm{pNF}-\mathrm{H} \text { CSF, pg/mL } \\
\mathrm{n}\end{array}$ & $\begin{array}{l}425(142-1,929) \\
67\end{array}$ & & $\begin{array}{l}540 \\
(174-1,929) \\
27\end{array}$ & & $\begin{array}{l}378(142-1,599) \\
26\end{array}$ & & $\begin{array}{l}442 \\
(142-1,929) \\
53\end{array}$ & & $\begin{array}{l}314(156-645) \\
14\end{array}$ & & $\begin{array}{l}201 \\
(129-533) \\
5\end{array}$ & \\
\hline $\begin{array}{l}\text { Tau CSF, pg/mL } \\
n\end{array}$ & $\begin{array}{l}353(102-1,422) \\
65\end{array}$ & & $\begin{array}{l}326 \\
(139-792) \\
26\end{array}$ & & $\begin{array}{l}343(102-785) \\
25\end{array}$ & & $\begin{array}{l}339 \\
(102-792) \\
51\end{array}$ & & $\begin{array}{l}703 \\
(171-1,422) \\
14\end{array}$ & & $\begin{array}{l}213 \\
(172-290) \\
5\end{array}$ & \\
\hline$\underset{n}{p \text {-tau }}$ CSF, pg/mL & $\begin{array}{l}49(16-191) \\
66\end{array}$ & & $\begin{array}{l}42(24-100) \\
26\end{array}$ & & $\begin{array}{l}48.5(16-89) \\
26\end{array}$ & & $\begin{array}{l}44.5(16-100) \\
52\end{array}$ & & $\begin{array}{l}88.5(25-191) \\
14\end{array}$ & & $\begin{array}{l}44(30-54) \\
5\end{array}$ & \\
\hline $\begin{array}{l}A \beta_{1-42} \mathrm{CSF}, \mathrm{pg} / \mathrm{mL} \\
\mathrm{n}\end{array}$ & $\begin{array}{l}757(312-1,601) \\
65\end{array}$ & & $\begin{array}{l}928 \\
(336-1,376) \\
26\end{array}$ & & $\begin{array}{l}761(312-1,601) \\
25\end{array}$ & & $\begin{array}{l}869 \\
(312-1,601) \\
51\end{array}$ & & $\begin{array}{l}552(314-952) \\
14\end{array}$ & & $\begin{array}{l}1,114 \\
(725-1,455) \\
5\end{array}$ & \\
\hline
\end{tabular}

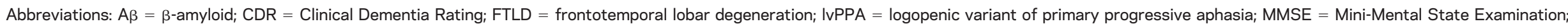

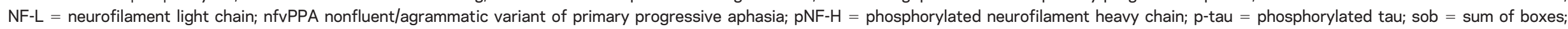
svPPA = semantic variant of primary progressive aphasia; v1 = baseline visit; v2 = follow-up visit.

Median values (ranges) are given.

${ }^{a}$ Age of patients with nfvPPA and svPPA is different for both the serum cohort and the CSF cohort $(p<0.01)$.

${ }^{b}$ One value missing.

${ }^{\mathrm{c}}$ Two values missing.

${ }^{\mathrm{d}}$ Three values missing. 
and for each PPA variant), nonparametric tests were applied throughout the analyses. To compare 2 datasets of marker concentrations, a 2-tailed unpaired Mann-Whitney $t$ test or Wilcoxon matched-pair test for analysis of longitudinal data was used. To analyze $>2$ datasets of marker concentrations, a Kruskal-Wallis test was used. In case of significance, a Dunn post hoc comparison correcting for multiple testing was applied. Correlation between the average levels of fluid analytes, neuropsychological rating scores, disease progression rates, or volumetric measures obtained from neuroimaging was calculated by the Spearman rank correlation coefficient. Level of significance was set at $p=0.05$.

The optimal cutoff level for dichotomizing values was selected as the situation maximizing the Youden index. The receiveroperating characteristics (ROC) curve is used for a graphic visualization of the effect of the variation in the cutoff values.

In the analysis of volumetric data, correlations with NF-L serum levels were determined, and coefficients $>0.5$ were regarded as positive if $p<0.05$. Here, the Bonferroni correction also was assessed (level of significance $p<0.002$ ). Additionally, linear regression analysis was applied to determine whether there was a linear association between 2 parameters showing significant Spearman correlation. Cross-sectional data obtained at v1 were corrected for intracranial volume. In analyses of longitudinal measures, original volumes were analyzed.

RESULTS Demographic and disease characteristics. Table 1 provides a summary of demographic characteristics of the PPA subgroups. For serum and CSF investigations, but not MRI, patients with nfvPPA were older at disease onset than patients with svPPA $(p<0.05)$. Disease duration was different in the PPA subgroups analyzed for serum NF-L $(p=0.038)$; however, the post hoc test failed to be significant. Disease severity at $\mathrm{v} 1$ and $\mathrm{v} 2$ or the functional decline during follow-up as quantified by means of CDR, FTLD-CDR, and MMSE score was not different between PPA groups for serum, CSF, or MRI investigations.

Degree of atrophy in cortical and subcortical brain areas was higher for svPPA compared to nfvPPA and lvPPA. While for lvPPA atrophy was more dominant in the left hemisphere, it was mostly symmetric for svPPA atrophy. For a summary of brain volumetric measures, see table e-1 and appendix e-1 at Neurology.org.

Serum NF-L at baseline visit and comparison to CSF markers. Table 1 gives median concentrations and range of markers. The serum NF-L level at first examination was higher in each of the PPA variants compared to the control group (figure 1A). Levels within the PPA cohort differed $(p=0.0007)$, with higher NF-L concentrations in nfvPPA and svPPA compared to lvPPA. ROC analysis yielded 95\% sensitivity and $70 \%$ specificity (cutoff $25 \mathrm{pg} / \mathrm{mL}$ ) for discrimination between PPA and controls and $81 \%$ sensitivity and $67 \%$ specificity (cutoff $31 \mathrm{pg} /$ $\mathrm{mL}$ ) for discrimination of pooled nfvPPA/svPPA and lvPPA (figure 1A).
All CSF markers showed different levels in the PPA variants (NF-L $p=0.0002$, pNF-H $p=$ 0.0022, $\mathrm{A} \beta_{1-42} p=0.0032$, tau $p=0.0093$, p-tau $p=0.0088)$. NF-L level was higher in nfvPPA and svPPA compared to lvPPA. By contrast, pNF-H was higher in nfvPPA compared to both svPPA and lvPPA (figure $1 \mathrm{C}$ ). $\mathrm{A} \beta_{1-42}$ was lower in lvPPA than in nfvPPA, with intermediate levels in svPPA (figure 1D). Tau and p-tau showed the highest levels in lvPPA, while in both nfvPPA and svPPA CSF samples, tau and p-tau were expressed at low levels (figure $1, \mathrm{E}$ and $\mathrm{F}$ ).

ROC analysis (curves in figure 1, B-E) revealed for CSF NF-L 92\% sensitivity and 78\% specificity for discrimination of lvPPA from nfvPPA/svPPA at a cutoff of 2,274 pg/mL. pNF-H yielded $84 \%$ sensitivity and $69 \%$ specificity (cutoff $494 \mathrm{pg} / \mathrm{mL}$ ) for differentiation between nfvPPA and svPPA. A $\beta_{1-42}$ allowed discrimination between lvPPA and nfvPPA/svPPA with $86 \%$ sensitivity and 69\% specificity (cutoff $705 \mathrm{pg} / \mathrm{mL}$ ). Detection of lvPPA was possible with a sensitivity of $84 \%$ for tau (cutoff $532 \mathrm{pg} / \mathrm{mL}$ ) and $90 \%$ for p-tau (cutoff $81 \mathrm{pg} / \mathrm{mL}$ ), with respective specificities of $71 \%$ and $57 \%$.

Correlation analysis of markers was conducted for all patients with PPA together and for each subgroup separately. Table e-2 gives a summary of results.

Serum NF-L at second examination after 1 year. Serum NF-L at follow-up examination correlated with serum NF-L determined at baseline (for all PPA $r=0.8132$, $p<0.0001)$ and showed higher concentrations for nfvPPA and svPPA compared to lvPPA and healthy controls. On average, levels of serum NF-L in patients with nfvPPA and svPPA increased from v1 to v2 $(p=$ 0.0018 and $p=0.0017$, respectively), while in lvPPA, the average NF-L level changed only marginally $(p=$ 0.4375 ) (figure 2).

NF-L and atrophy at baseline examination. Table e-3 provides a summary of statistical results. In lvPPA, high NF-L concentration correlated with a low volume of the right temporal lobe $(r=-0.8214$, $p=0.0234)$ and the left and right middle temporal gyrus, the right inferior temporal gyrus, and the left fusiform gyrus. None of the results survived correction for multiple comparisons. For nfvPPA and svPPA, there was no correlation of NF-L with the volume of any area.

NF-L as a predictor of brain atrophy. High serum NF-L of patients with svPPA correlated with stronger progression of atrophy in the left middle orbitofrontal gyrus $(r=-0.7619, p=0.028)$. Level of significance for data corrected for multiple comparisons was not reached, and the association was not linear $\left(r^{2}=0.2403, p=0.2175\right)$. 

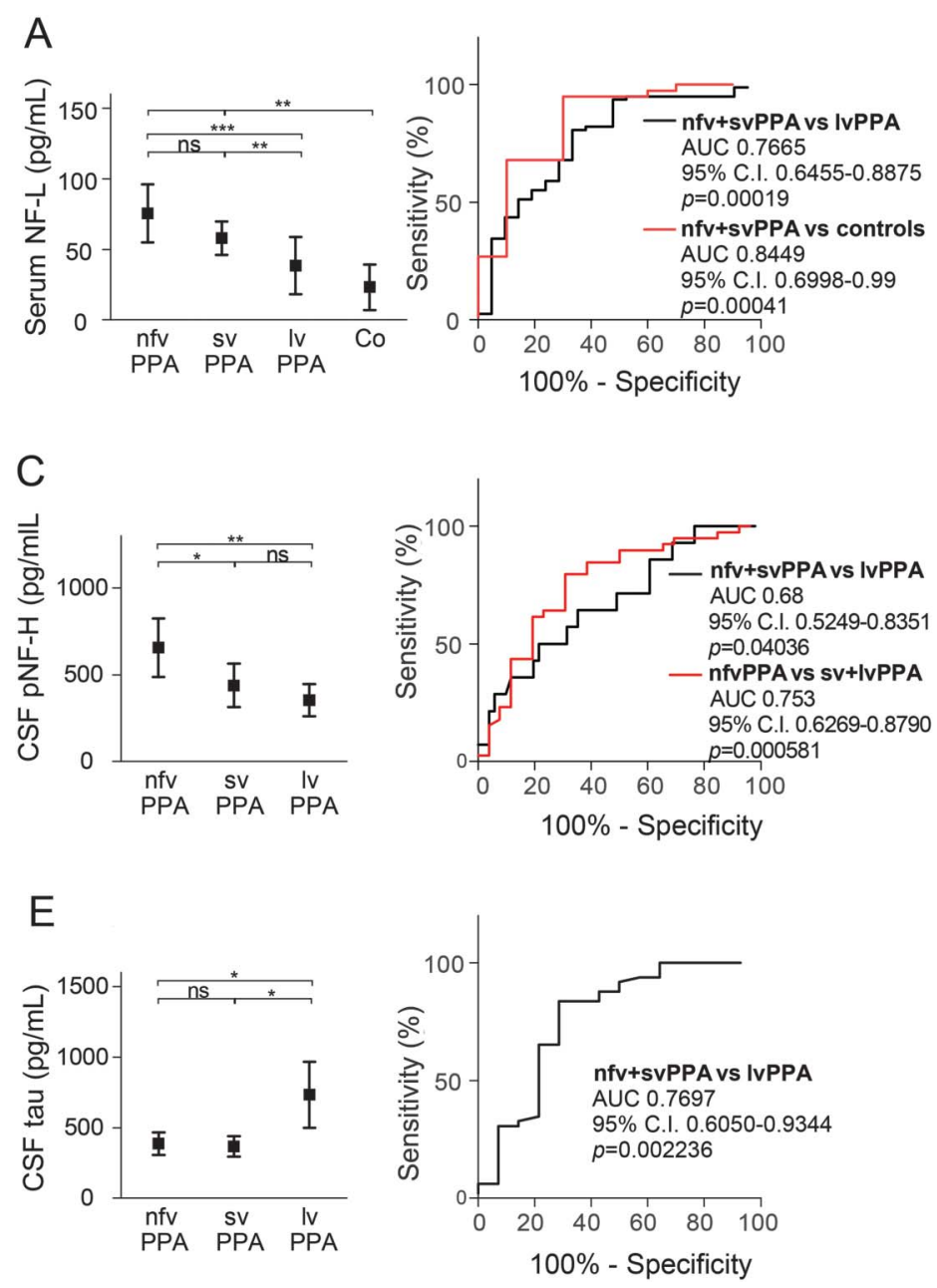
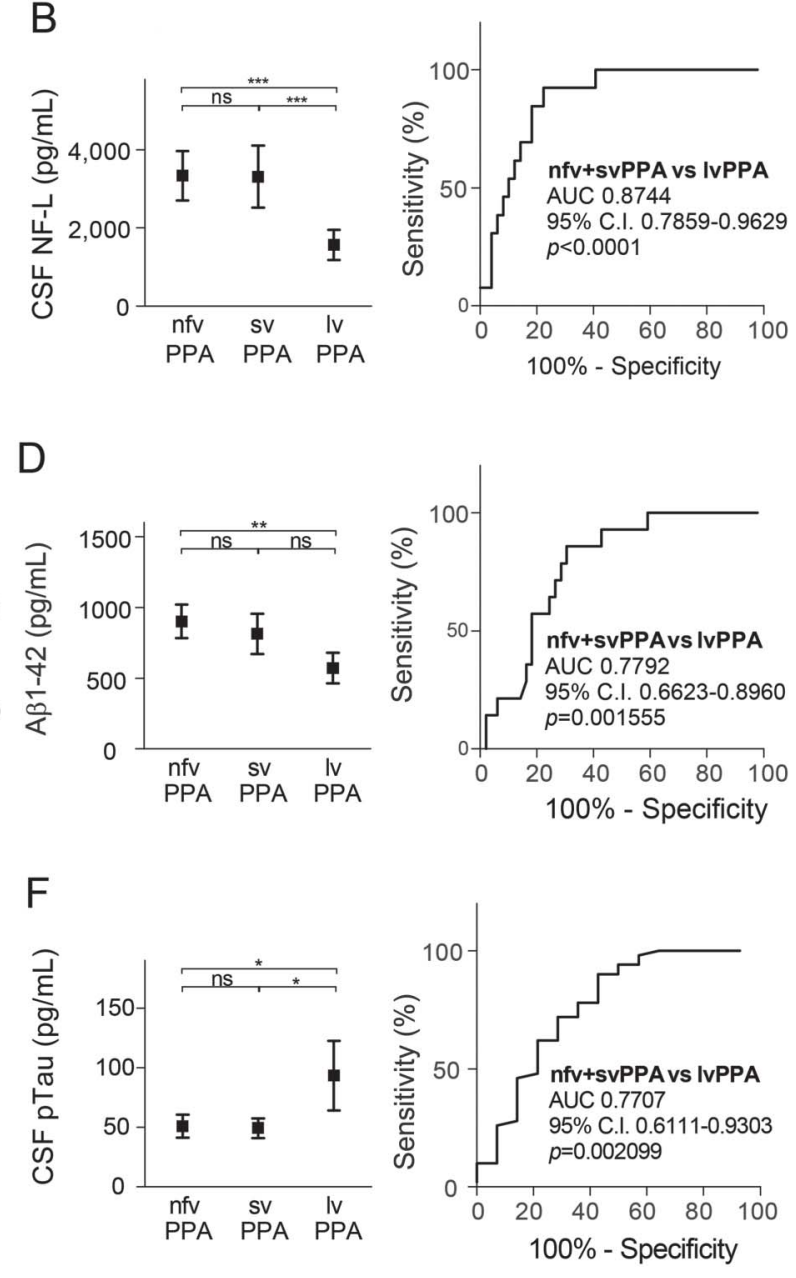

The neurofilament light chain (NF-L) levels in nonfluent/agrammatic variant (nfvPPA), semantic variant (svPPA), and logopenic variant (lvPPA) of primary progressive aphasia serum samples and in control (Co) samples from healthy volunteers are shown by box plots, and the resulting diagnostic performance is indicated by receiver-operating characteristics (ROC) curves with respective statistical values (A). Levels and resulting ROC curve of CSF NF-L (B), phosphorylated neurofilament heavy chain (pNF-H; $C)$, $\beta$-amyloid $\left(A \beta_{1-42} ; D\right)$, tau $(E)$, and phosphorylated tau $(p T a u ; F)$ are given for PPA variants. Boxes show the mean concentration; whiskers indicate $95 \%$ confidence interval (C.I.). Kruskal-Wallis test for statistical analysis of the 3 PPA groups revealed differences for all markers. Results of the respective post hoc tests are indicated by asterisks (*p $\left.<0.05,{ }^{* *} p<0.01, * * * p 0.001\right)$. AUC $=$ area under the curve; ns = not significant.

Serum NF-L and brain atrophy in the course of disease. Remarkably, progression of atrophy in several brain areas correlated with the serum NF-L course in the analysis of all patients with PPA and the PPA subgroups (table e-3). In patients with marked increase of NF-L, the progression of atrophy of the left frontal lobe (Spearman $r=-0.6375, p=$ 0.0014 ) and of the right frontal lobe (Spearman $r=$ $-0.5539, p=0.0075$ ) (figure 3) was most pronounced. Furthermore, the more NF-L increased, the stronger the atrophy of the right middle frontal gyrus and the left gyrus rectus was. When looking at all patients with PPA, correlation of NF-L increase and atrophy progression of the left frontal lobe survived correction for multiple comparisons. Similarly, when looking at patients with nfvPPA and svPPA, the same holds true for the right middle frontal gyrus.
Correlation of baseline serum NF-L with disease duration, functional assessment, and progression rates. Serum NF-L levels only weakly correlated with the age of all patients with PPA at disease onset $(r=$ 0.233, $p=0.026$ ) and not with disease duration $(r=-0.228, p=0.228)$. In the subgroup analysis, there was no correlation for nfvPPA or svPPA of serum NF-L with age or disease duration, but we found a correlation for the age at onset with NF-L in the lvPPA cohort $(r=0.5, p=0.029)$.

Serum NF-L levels did not correlate with CDR, FTLD-CDR, or MMSE In addition, for the progression rates at baseline calculated on the basis of the functional scores, we found no correlation with serum NF-L levels except for a correlation between NF-L and MMSE progression rate at baseline examination in the lvPPA group $(r=0.47, p=0.049)$. 
Figure 2 Serum NF-L concentrations at follow-up examination of the 3 PPA variants and healthy controls
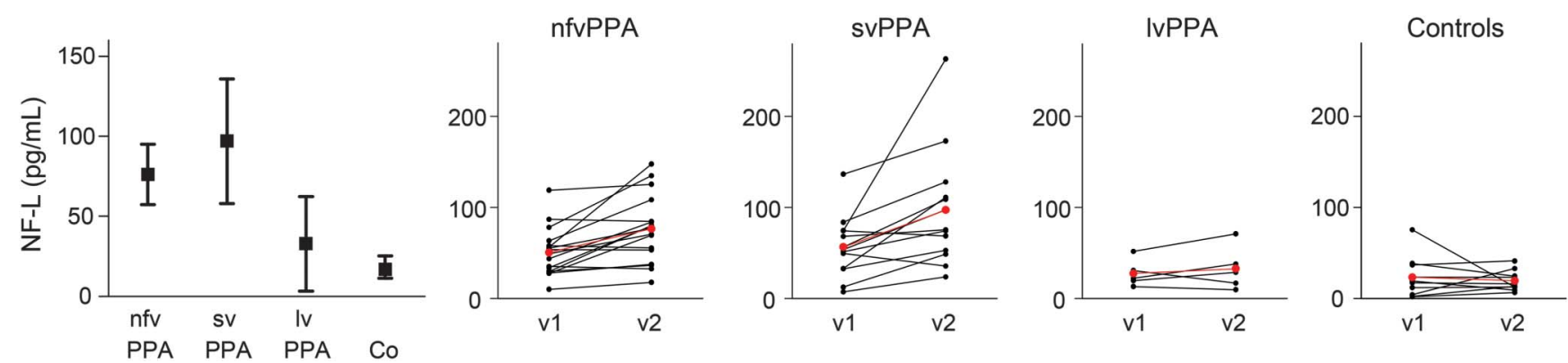

Boxes show the mean concentration; whiskers indicate $95 \%$ confidence interval (C.I.). Individual courses for nonfluent/agrammatic variant (nfvPPA), semantic variant (svPPA), and logopenic variant (IvPPA) of primary progressive aphasia and healthy controls (Co) of the neurofilament light chain (NF-L) concentration in serum at baseline visit (v1) and 1 year later (v2). Mean courses are shown by red lines and symbols.

\section{Figure 3 Serum NF-L change and progression of brain atrophy from baseline to follow-up visit}

A. Left frontal lobe of PPA patients

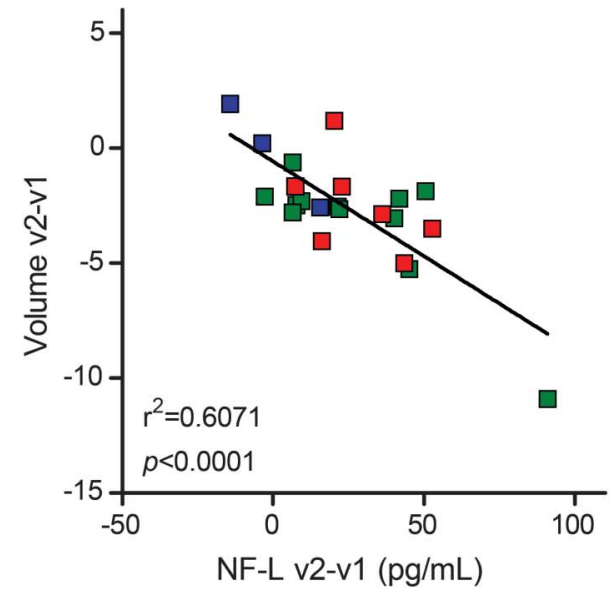

C. Left frontal lobe of healthy patients

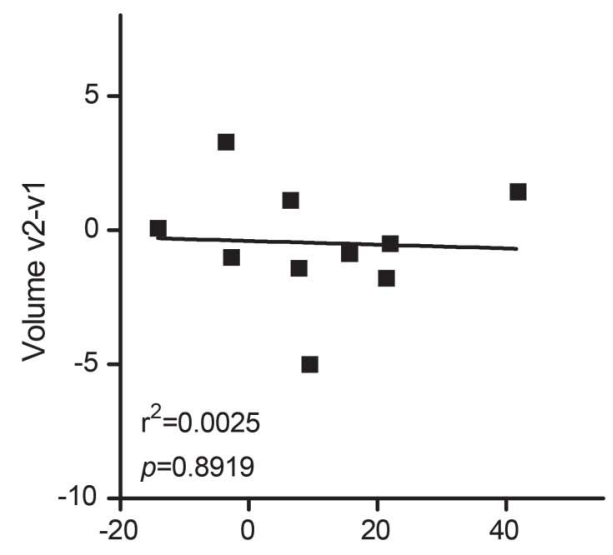

NF-L v2-v1 $(\mathrm{pg} / \mathrm{mL})$

\section{B. Right frontal lobe of PPA patient}

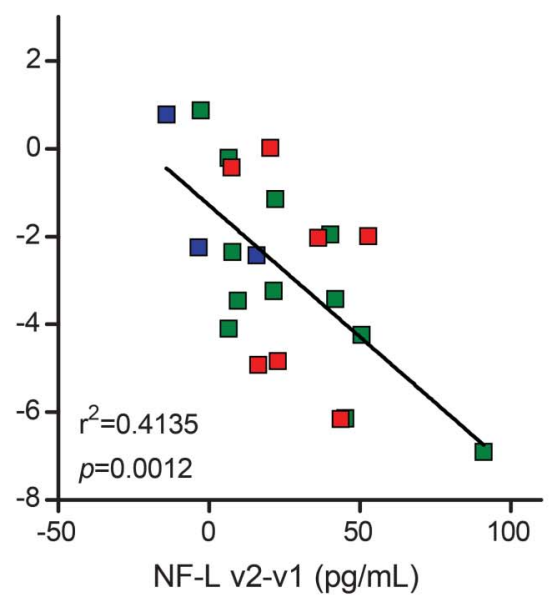

D. Right frontal lobe of healthy patients

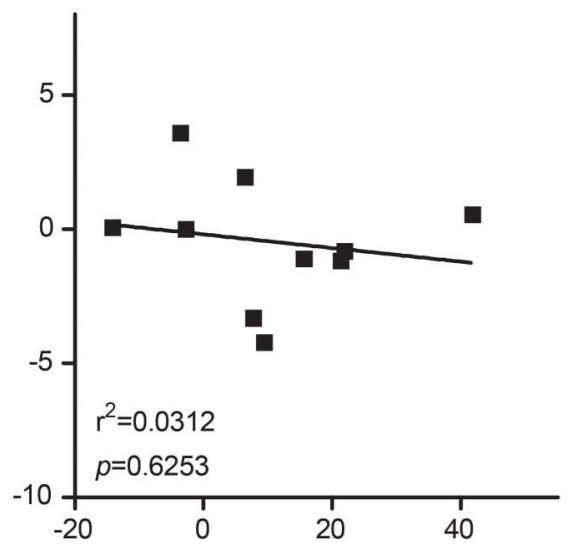

NF-L v2-v1 $(p g / m L)$

Colors indicate the primary progressive aphasia (PPA) variant: nonfluent/agrammatic variant (nfvPPA) in green, semantic variant (svPPA) in red, and logopenic variant (IVPPA) in blue. (A) Left frontal lobe of patients with PPA, (B) right frontal lobe of patients with PPA, (C) left frontal lobe of healthy participants, and (D) right front lobe of healthy participants. The results of linear regression are given in the respective graphs and are indicated by lines. NF-L = neurofilament light chain; $\mathrm{v} 1=$ baseline visit; v2 = follow-up visit 1 year later. 

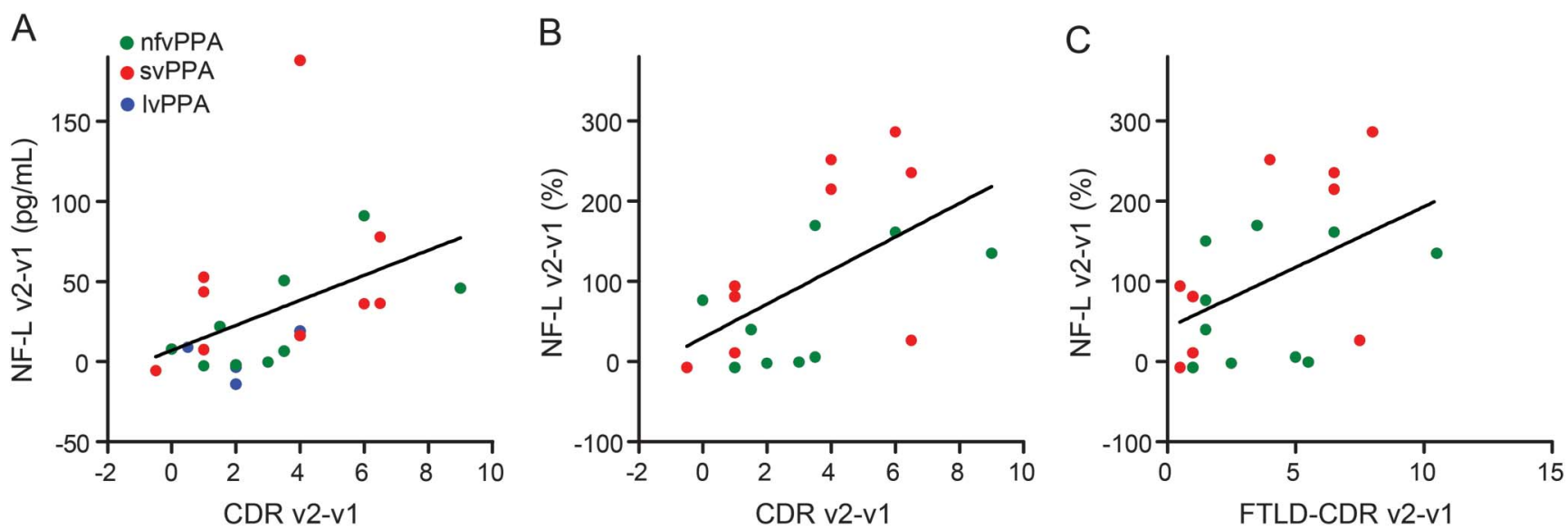

The increase in the Clinical Dementia Rating (CDR) score vs the absolute change of neurofilament light chain (NF-L) measured in serum samples of all patients with primary progressive aphasia (PPA; A) and vs the relative change in serum NF-L level of patients with the nonfluent/agrammatic variant (nfvPPA) and semantic variant (svPPA; B) is shown. (C) Relationship of the serum NF-L change and the increase in the frontotemporal lobar degeneration (FTLD)-CDR score during follow-up is illustrated. Lines show the linear regression ( $A: r^{2}=0.1888, p=0.0433 ; B: r^{2}=0.3108, p=0.0162 ; C: r^{2}=0.2196, p=0.043$ ). IvPPA = logopenic variant; v1 = baseline visit; v2 = follow-up visit 1 year later.

Serum NF-L and functional decline during follow-up. We analyzed whether serum NF-L levels at baseline or the change in serum NF-L from baseline to follow-up is indicative of disease progression assigned by neuropsychological assessment during follow-up (figure 4). For the entire PPA cohort, there was a correlation of the absolute or normalized NF-L change and the disease progression rate as determined by CDR (absolute NF-L change: $r=0.524, p=0.012$; relative NF-L change: $r=0.485, p=0.022$ ) (figure 4, A and B). Analyzing nfvPPA and svPPA (the groups with increasing NF-L levels during follow-up) together revealed a correlation between not only the relative NF-L change and CDR progression rate $(r=0.594$, $p=0.009)$ but also the FTLD-CDR progression rate $(r=0.496, p=0.031)$ (figure $4 \mathrm{C})$.

DISCUSSION Diagnosis of clinical PPA variants according to the consensus criteria leads to a significant number of unclassified cases, ${ }^{22,23}$ and objective biomarkers are needed. Fluid biomarker research has focused on CSF to discriminate between FTLD subgroups, ${ }^{3-5,24}$ which seems rational because CSF directly contacts the central nervous system, thus representing the most promising source to reflect neurodegenerative processes. ${ }^{25}$ Increased levels of CSF NF-L in svPPA and nfvPPA and a pattern of $A \beta_{1-42}$, tau, and p-tau comparable to that characteristic for Alzheimer disease in lvPPA have been reported, ${ }^{4,24,26}$ which is in accordance with our results. Thus, we provide further evidence for these markers to be suitable for PPA subgroup differentiation.

In our cohort, pNF-H was the only marker able to discriminate nfvPPA from sv/lvPPA, which has not been described before. If this result can be confirmed in a validation cohort or even in serum, pNF-H represents a good candidate to improve PPA variant discrimination in combination with markers discriminating nfvPPA/svPPA from lvPPA. Here, NF-L seems to be the superior candidate.

Recent technical advances have made it possible to measure brain-derived proteins in the blood that indicate neurodegeneration, including NF-L. ${ }^{21}$ Consequently, it could be shown that blood markers may be suitable for supporting the diagnosis and for prognosis of disease progression. ${ }^{20,27-30}$

We found higher NF-L levels in nfvPPA and svPPA than in lvPPA, and longitudinally, an NF-L increase indicates disease severity: the more pronounced progression of atrophy was and the faster the patients' functional performance declined, the more serum NF-L increased. A recent study also reported higher serum NF-L in nfvPPA and svPPA and determined higher levels in patients with frontotemporal dementia, including 11 patients with PPA, correlating with higher annual atrophy rates after serum sampling. ${ }^{6}$ Within the cohort we analyzed, NF-L at baseline predicted the degree of left middle orbitofrontal gyrus atrophy of patients with svPPA. A more widespread pattern of correlation was seen for NF-L increase and progression of atrophy, which we observed predominantly for the left hemisphere, consistent with the characteristic pattern of atrophy in PPA, especially nfvPPA. ${ }^{31,32}$ Additionally, in a considerable number of the brain areas analyzed, NF-L also correlated with right-sided atrophy, which could be explained by impaired compensation mechanisms underlying disease progression. Looking at an association between NF-L and functional performance, we found no correlation at baseline but 
a correlation of the NF-L increase with CDR score increase. Here, it would be interesting to see whether NF-L also correlates with specific domains or functions. Taken together, analysis of longitudinal data provides evidence for the usefulness of serum NF-L as a PPA disease status marker correlating with symptom and atrophy exacerbation rather than as a prognostic marker. It would be important for a monitoring marker that the level correlates with disease parameters from the onset. A cross-sectional study of patients with amyotrophic lateral sclerosis, a neurodegenerative disease genetically and pathophysiologically associated with FTLD, indicates that NF-L increases in parallel to symptom appearance. ${ }^{29}$ A similar study on patients with FTLD is currently pending.

A diagnostic and prognostic value of CSF NF-L levels for amyotrophic lateral sclerosis was reported, ${ }^{29,33,34}$ and 2 recent publications on longitudinal serum NF-L showed that the level mostly stays stable during follow-up. ${ }^{32,33}$ This points to a different influence of either the localization of neurodegeneration in the CNS or the rate of neurodegeneration on the NF-L serum level.

Whether the molecular pathologic characteristics of PPA variants prominently affect the NF-L level remains unclear. In an examination of mouse models of neurodegenerative diseases, it has recently been shown that CSF and plasma NF-L levels were increased, correlating with the proteopathic lesions (A $\beta, \alpha$-synuclein, tau). ${ }^{35}$ Studies examining FTLDTAR DNA-binding protein and FTLD-tau CSF samples provide evidence that TAR DNA-binding protein-43 pathology is associated with higher NF-L levels. ${ }^{3,4,24}$ We found similar NF-L levels in the CSF and a trend for higher serum NF-L levels in the serum of nfvPPA compared to svPPA. It has to be considered that PPA variants show a prominent overlap of pathology ${ }^{1,36,37}$ and that fulfillment of certain clinical criteria is indicative of an underlying pathology only at the group level. ${ }^{38}$ Therefore, only analysis of large PPA cohorts comprising neuropathologically characterized cases will answer this question. Here, preferably single-molecule array technique might be used because it currently is the most sensitive method. ${ }^{21}$

There are several limitations associated with our study. Because it is a multicenter study, additional variances in the volumetric measurements can be assumed, ${ }^{8}$ and some datasets lack completeness; e.g., not all patients were genetically analyzed, and for some, the second neuropsychological assessments were missing. Reasons for the latter are diverse, and an influence on serum follow-up data cannot be excluded.

Future studies of NF-L in serum samples repeatedly collected over a longer period and parallel longitudinal characterization by MRI and neuropsychological testing will clarify whether NF-L reflects atrophy in specific brain areas more than in others and correlates with distinctive neuropsychological symptoms.

\section{AUTHOR CONTRIBUTIONS}

Study conception and design: P.S., M.O., J.D.-S., A.D., A.C.L. Acquisition and analysis of data: P.S., E.S., S.A.-S., M.L.S., I.U., H.F., B.L., C.A.F.v.A., J.K., P.O., H.-J.H., K.F., K.F., J.P., C.R., J.K., A.S., A.E.V., M.L., A.D., A.C.L., M.O. Writing of the manuscript: P.S., M.O., M.L.S., H.-J.H. Critical revision of the manuscript: P.S., E.S., S.A.-S., J.D.-S., M.L.S., I.U., H.F., B.L., C.A.F.v.A., J.K., P.O., H.-J.H., K.F., K.F., J.P., C.R., J.K., A.S., A.E.V., M.L., A.D., A.C.L., M.O.

\section{ACKNOWLEDGMENT}

The authors thank all patients for participating in this study.

\section{STUDY FUNDING}

The study was supported by grants from the German Federal Ministry of Education and Research (project FTLDc 01GI1007A, MND-Net 01GI0704); the EU Joint Programme-Neurodegenerative Diseases networks SOPHIA (01ED1202A), BiomarkAPD (01ED1203F), and PreFrontAls (01ED1512); and the foundation of the state of Baden-Württemberg (D.3830) and BIU (D.5009). Furthermore, M.L.S. acknowledges support by the Parkinson's Disease Foundation (grant No. PDF-IRG-1307) and by the Michael Fox Foundation (grant No. 11362).

\section{DISCLOSURE}

The authors report no disclosures relevant to the manuscript. Go to Neurology.org for full disclosures.

Received July 27, 2016. Accepted in final form December 14, 2016.

\section{REFERENCES}

1. Gorno-Tempini ML, Hillis AE, Weintraub S, et al. Classification of primary progressive aphasia and its variants. Neurology 2011;76:1006-1014.

2. Grossman M. Biomarkers in the primary progressive aphasias. Aphasiology 2014;28:922-940.

3. Pijnenburg YA, Verwey NA, van der Flier WM, Scheltens P, Teunissen CE. Discriminative and prognostic potential of cerebrospinal fluid phosphoTau/tau ratio and neurofilaments for frontotemporal dementia subtypes. Alzheimers Dement (Amst) 2015;1:505-512.

4. Scherling CS, Hall T, Berisha F, et al. Cerebrospinal fluid neurofilament concentration reflects disease severity in frontotemporal degeneration. Ann Neurol 2014;75:116-126.

5. Skillback T, Farahmand B, Bartlett JW, et al. CSF neurofilament light differs in neurodegenerative diseases and predicts severity and survival. Neurology 2014;83: 1945-1953.

6. Rohrer JD, Woollacott IO, Dick KM, et al. Serum neurofilament light chain protein is a measure of disease intensity in frontotemporal dementia. Neurology 2016;87:1329-1336.

7. Otto M, Ludolph AC, Landwehrmeyer B, et al. German consortium for frontotemporal lobar degeneration [in German]. Nervenarzt 2011;82:1002-1005.

8. Teipel S, Raiser T, Riedl L, et al. Atrophy and structural covariance of the cholinergic basal forebrain in primary progressive aphasia. Cortex 2016;83:124-135.

9. Folstein MF, Folstein SE, McHugh PR. "Mini-mental state": a practical method for grading the cognitive state of patients for the clinician. J Psychiatr Res 1975;12: 189-198.

10. Morris JC. The Clinical Dementia Rating (CDR): current version and scoring rules. Neurology 1993;43:2412-2414. 
11. Knopman DS, Kramer JH, Boeve BF, et al. Development of methodology for conducting clinical trials in frontotemporal lobar degeneration. Brain 2008;131:2957-2968.

12. Huppertz HJ, Kroll-Seger J, Kloppel S, Ganz RE, Kassubek J. Intra- and interscanner variability of automated voxel-based volumetry based on a $3 \mathrm{D}$ probabilistic atlas of human cerebral structures. Neuroimage 2010;49:2216-2224.

13. Huppertz HJ, Moller L, Sudmeyer M, et al. Differentiation of neurodegenerative parkinsonian syndromes by volumetric magnetic resonance imaging analysis and support vector machine classification. Mov Disord 2016;31: 1506-1517.

14. Shattuck DW, Mirza M, Adisetiyo V, et al. Construction of a 3D probabilistic atlas of human cortical structures. Neuroimage 2008;39:1064-1080.

15. Frazier JA, Chiu S, Breeze JL, et al. Structural brain magnetic resonance imaging of limbic and thalamic volumes in pediatric bipolar disorder. Am J Psychiatry 2005;162: 1256-1265.

16. Frings L, Mader I, Landwehrmeyer BG, Weiller C, Hull M, Huppertz HJ. Quantifying change in individual subjects affected by frontotemporal lobar degeneration using automated longitudinal MRI volumetry. Hum Brain Mapp 2012;33:1526-1535.

17. Kassubek J, Pinkhardt EH, Dietmaier A, Ludolph AC, Landwehrmeyer GB, Huppertz HJ. Fully automated atlasbased MR imaging volumetry in Huntington disease, compared with manual volumetry. AJNR Am J Neuroradiol 2011;32:1328-1332.

18. Opfer R, Suppa P, Kepp T, Spies L, Schippling S, Huppertz HJ. Atlas based brain volumetry: how to distinguish regional volume changes due to biological or physiological effects from inherent noise of the methodology. Magn Reson Imaging 2016;34:455-461.

19. Bisenius S, Neumann J, Schroeter ML. Validating new diagnostic imaging criteria for primary progressive aphasia via anatomical likelihood estimation meta-analyses. Eur J Neurol 2016;23:704-712.

20. Gaiottino J, Norgren N, Dobson R, et al. Increased neurofilament light chain blood levels in neurodegenerative neurological diseases. PLoS One 2013;8:e75091.

21. Kuhle J, Barro C, Andreasson U, et al. Comparison of three analytical platforms for quantification of the neurofilament light chain in blood samples: ELISA, electrochemiluminescence immunoassay and Simoa. Clin Chem Lab Med 2016;54:1655-1661.

22. Wicklund MR, Duffy JR, Strand EA, Machulda MM, Whitwell JL, Josephs KA. Quantitative application of the primary progressive aphasia consensus criteria. Neurology 2014;82:1119-1126.
23. Sajjadi SA, Patterson K, Arnold RJ, Watson PC, Nestor PJ. Primary progressive aphasia: a tale of two syndromes and the rest. Neurology 2012;78:1670-1677.

24. Landquist Waldo M, Frizell Santillo A, Passant U, et al. Cerebrospinal fluid neurofilament light chain protein levels in subtypes of frontotemporal dementia. BMC Neurol 2013;13:54.

25. Oeckl P, Steinacker P, Feneberg E, Otto M. Neurochemical biomarkers in the diagnosis of frontotemporal lobar degeneration: an update. J Neurochem 2016;138(suppl 1):184-192.

26. Santangelo R, Coppi E, Ferrari L, et al. Cerebrospinal fluid biomarkers can play a pivotal role in the diagnostic work up of primary progressive aphasia. J Alzheimers Dis 2015; 43:1429-1440.

27. Noguchi-Shinohara M, Hamaguchi T, Nozaki I, Sakai K, Yamada M. Serum tau protein as a marker for the diagnosis of Creutzfeldt-Jakob disease. J Neurol 2011;258:1464-1468.

28. Oliver JM, Jones MT, Kirk KM, et al. Serum neurofilament light in American football athletes over the course of a season. J Neurotrauma 2016;33:1784-1789.

29. Weydt P, Oeckl P, Huss A, et al. Neurofilament levels as biomarkers in asymptomatic and symptomatic familial amyotrophic lateral sclerosis. Ann Neurol 2016;79:152-158.

30. Zetterberg H, Wilson D, Andreasson U, et al. Plasma tau levels in Alzheimer's disease. Alzheimers Res Ther 2013;5:9.

31. Grossman M, Mickanin J, Onishi K, et al. Progressive nonfluent aphasia: language, cognitive, and PET measures contrasted with probable Alzheimer's disease. J Cogn Neurosci 1996;8:135-154.

32. Josephs KA, Duffy JR, Strand EA, et al. Clinicopathological and imaging correlates of progressive aphasia and apraxia of speech. Brain 2006;129:1385-1398.

33. Lu CH, Macdonald-Wallis C, Gray E, et al. Neurofilament light chain: a prognostic biomarker in amyotrophic lateral sclerosis. Neurology 2015;84:2247-2257.

34. Steinacker P, Feneberg E, Weishaupt J, et al. Neurofilaments in the diagnosis of motoneuron diseases: a prospective study on 455 patients. J Neurol Neurosurg Psychiatry 2016;87:12-20.

35. Bacioglu M, Maia LF, Preische O, et al. Neurofilament light chain in blood and CSF as marker of disease progression in mouse models and in neurodegenerative diseases. Neuron 2016;91:56-66.

36. Grossman M. Primary progressive aphasia: clinicopathological correlations. Nat Rev Neurol 2010;6:88-97.

37. Josephs KA, Hodges JR, Snowden JS, et al. Neuropathological background of phenotypical variability in frontotemporal dementia. Acta Neuropathol 2011;122:137-153.

38. Hu WT, McMillan C, Libon D, et al. Multimodal predictors for Alzheimer disease in nonfluent primary progressive aphasia. Neurology 2010;75:595-602. 